\title{
Some new models of strange stars in 5-D Einstein-Gauss-Bonnet Gravity
}

\author{
Manuel Malaver ${ }^{1,2}$ and Hamed Daei Kasmaei ${ }^{3}$ \\ ${ }^{1}$ Bijective Physics Institute, Idrija, Slovenia \\ ${ }^{2}$ Maritime University of the Caribbean, Department of Basic Sciences, Catia la Mar, Venezuela. \\ Email: mmf.umc@gmail.com \\ ${ }^{3}$ Islamic Azad University, Department of Applied Mathematics and Computer Science, Central Tehran \\ Branch, Tehran, Iran \\ Email: hamedelectroj@gmail.com
}

\begin{abstract}
In this paper, we present some new models for anisotropic compact stars within the framework of 5-dimensional Einstein-Gauss-Bonnet (EGB) gravity with a linear and nonlinear equation of state considering a metric potential proposed for Thirukkanesh and Ragel (2012) and generalized for Malaver (2014). The new obtained models satisfy all physical requirements of a physically reasonable stellar object. Variables as energy density, radial pressure and the anisotropy are dependent of the values of the Gauss-Bonnet coupling constant.
\end{abstract}

Keywords: EGB Gravity, Compact Stars, Equation of State, Metric Potential, Coupling Constant

\section{Introduction}

Mathematical modeling within the framework of the general theory of relativity has been used to explain the behavior and structure of massive objects as neutron stars, quasars, black holes, pulsars and white dwarfs [1,2] and requires finding the exact solutions of the Einstein-Maxwell system [3]. A detailed and systematic analysis was carried out by Delgaty and Lake [4] which obtained several analytical solutions that can describe realistic stellar configurations.

It is very important to mention the pioneering works of Schwarzschild [5], Tolman [6], Oppenheimer and Volkoff [7] and Chandrasekhar [8] in the development of the first theoretical models of stellar objects. Schwarzschild [5] obtained interior solutions that allows describing a star with uniform density, Tolman [6] generated new solutions for static spheres of fluid, Oppenheimer and Volkoff [7] studied the gravitational equilibrium of neutron masses using the equation of state for a cold Fermi gas and general relativity and Chandrasekhar [8] produced new models of white dwarfs in presence of relativistic effects. Some of these results have been extended to higher dimensions and the dimensionality of space-time apparently influence the stability of these fluid spheres [9].

Recently, astronomical observations of compact objects have allowed new findings of neutron stars and strange stars that adjust to the exact solutions of the 4-D Einstein field equations and the data on mass maximum, redshift and luminosity are some of the most 
relevant characteristics for verifying the physical requirements of these models [10]. A great number of exact models from the Einstein-Maxwell field equations have been generated by Gupta and Maurya [11], Kiess [12], Mafa Takisa and Maharaj [13], Malaver and Kasmaei [14], Malaver [15,16], Ivanov [17] and Sunzu et al [18]. In the development of these models, several forms of equations of state can be considered [19]. Komathiraj and Maharaj [20], Malaver [21], Bombaci [22], Thirukkanesh and Maharaj [23], Dey et al. [24] and Usov [25] assume linear equation of state for quark stars. Feroze and Siddiqui [26] considered a quadratic equation of state for the matter distribution and specified particular forms for the gravitational potential and electric field intensity. MafaTakisa and Maharaj [13] obtained new exact solutions to the Einstein-Maxwell system of equations with a polytropic equation of state. Thirukkanesh and Ragel [27] have obtained particular models of anisotropic fluids with polytropic equation of state which are consistent with the reported experimental observations. Malaver [28] generated new exact solutions to the EinsteinMaxwell system considering Van der Waals modified equation of state with polytropic exponent. Tello-Ortiz et al. [29] found an anisotropic fluid sphere solution of the EinsteinMaxwell field equations with a modified version of the Chaplygin equation of state.

The analysis of compact objects with anisotropic matter distribution is very important, because that the anisotropy plays a significant role in the studies of relativistic spheres of fluid [30-42]. Anisotropy is defined as $\Delta=p_{t}-p_{r}$ where $p_{r}$ is the radial pressure and $p_{t}$ is the tangential pressure. The existence of solid core, presence of type 3A superfluid [43], magnetic field, phase transitions, a pion condensation and electric field [25] are most important reasonable facts that explain the presence of tangential pressures within a star. Many astrophysical objects as X-ray pulsar, Her X-1, 4U1820-30 and SAXJ1804.4-3658 have anisotropic pressures. Bowers and Liang [42] include in the equation of hydrostatic equilibrium the case of local anisotropy. Bhar et al. [44] have studied the behavior of relativistic objects with locally anisotropic matter distribution considering the Tolman VII form for the gravitational potential with a linear relation between the energy density and the radial pressure. Malaver [45-46], Feroze and Siddiqui [26,47] and Sunzu et al.[18] obtained solutions of the Einstein-Maxwell field equations for charged spherically symmetric space-time by assuming anisotropic pressure.

The behavior and dynamics of the gravitational field can be extended to higher dimensions [48]. The history of higher dimensions goes back to the work done by Kaluza [49] and Klein [50] who introduced the concept of extra dimensions in addition to the usual four dimensions (4-D) to unify gravitational and electromagnetic interactions. In general theory of relativity, the results obtained in four dimensions can be generalized in higher dimensional context and study the effects due to incorporation of extra space-time dimensions [51]. Within this framework, a very useful and fruitful generalization is the Einstein-Gauss-Bonnet gravity, which has generated a lot of interest among researchers and has been influenced by many scientists working in this field [52]. The modeling of compact objects in EGB gravity has shown that some physical variables are modified when they are compared to their 4-D counterparts, but the condition of the Schwarzschild constant density 
sphere has been demonstrated in EGB gravity [10]. Recently, Bhar et al. [53] performed a comparative study of compact objects in five dimensions (5-D) between EGB gravity and classical general relativity theory and found that many features as stability, causality and energy conditions remain unaffected in the stellar interior.

In this work, we have used the Thirukkanesh-Ragel-Malaver ansatz [27,37,54] in order to generate some stellar models with anisotropic matter distribution in EGB gravity. The system of field equations has been solved to obtain analytic solutions which are physically acceptable. The paper is organized as follows: In Section.2, we present the framework of EGB gravity. The modified Einstein-Maxwell field equations with the Gauss-Bonnet coupling constant are presented in Section.3. With the Thirukkanesh-Ragel-Malaver ansatz, we generate some models of an anisotropic star with a linear and nonlinear equation of state within EGB gravity in Section.4. In Section. 5, physical requirements for the new models are described. In Section.6, a physical analysis of the new solutions is performed. In final Section, we conclude.

\section{Einstein-Gauss-Bonnet Gravity}

The Gauss-Bonnet action in five dimensions can be written as

$$
S=\int \sqrt{-g}\left[\frac{1}{2}\left(R+\alpha L_{G B}\right)\right] d^{5} x+S_{\text {matter }}
$$

where $\alpha$ is the Gauss-Bonnet coupling constant. The strength of the action $L_{G B}$ lies in the fact that despite the Lagrangian being quadratic in the Ricci tensor, Ricci scalar and the Riemann tensor, the equations of motion turn out to be second order quasi-linear which are compatible with Einstein's theory of gravity $[52,53]$.

The EGB field equations may be written as

$$
G_{a b}+\alpha H_{a b}=T_{a b}
$$

where $G_{a b}$ represents the Einstein tensor, $T_{a b}$ is the total energy-momentum tensor and the Lanczos tensor $H_{a b}$ is given by

$$
H_{a b}=2\left(R R_{a b}-2 R_{a c} R_{b}^{c}-2 R^{c d} R_{a c b d}+R_{a}^{c d e} R_{b c d e}\right)-\frac{1}{2} g_{a b} L_{G B}
$$

where the Lovelock term has the form

$$
L_{G B}=R^{2}+R_{a b c d} R^{a b c d}-4 R_{c d} R^{c d}
$$




\section{Field Equations}

The 5-dimensional line element for a static spherically symmetric space-time takes the form

$$
d s^{2}=-e^{2 v(r)} d t^{2}+e^{2 \lambda(r)} d r^{2}+r^{2}\left(d \vartheta^{2}+\sin ^{2} \vartheta d \phi^{2}+\sin ^{2} \theta \sin ^{2} \phi d \psi^{2}\right)
$$

where the metric functions $e^{v}$ and $e^{\lambda}$ are the gravitational potentials. By considering the commoving fluid velocity as $u^{a}=e^{-v} \delta_{0}^{a}$, the EGB field equations (2) reduce to

$$
\begin{gathered}
\rho=\frac{3}{e^{4 \lambda} r^{3}}\left(4 \alpha \lambda^{\prime}+r e^{2 \lambda}-r e^{4 \lambda}-r^{2} e^{2 \lambda} \lambda^{\prime}-4 \alpha e^{2 \lambda} \lambda^{\prime}\right) \\
p_{r}=\frac{3}{e^{4 \lambda} r^{3}}\left(-r e^{4 \lambda}+\left(r^{2} v^{\prime}+r+4 \alpha v^{\prime}\right) e^{2 \lambda}-4 \alpha v^{\prime}\right) \\
\frac{1}{p_{t}}=\frac{1}{e^{4 \lambda} r^{2}}\left(-e^{4 \lambda}-4 \alpha v^{\prime \prime}+12 \alpha v^{\prime} \lambda^{\prime}-4 \alpha\left(v^{\prime}\right)^{2}\right)+ \\
\frac{1}{e^{2 \lambda} r^{2}}\left(1-r^{2} v^{\prime} \lambda^{\prime}+2 r v^{\prime}-2 r \lambda^{\prime}+r^{2}\left(v^{\prime}\right)^{2}\right)+ \\
\frac{1}{e^{2 \lambda} r^{2}}\left(r^{2} v^{\prime \prime}-4 \alpha v^{\prime} \lambda^{\prime}+4 \alpha\left(v^{\prime}\right)^{2}+4 \alpha v^{\prime \prime}\right)
\end{gathered}
$$

Here primes means a derivation with respect to the radial coordinates $r$ and $\rho$ is the energy density, $p_{r}$ is the radial pressure and $p_{t}$ is the tangential pressure. With the transformations $x=c r^{2}, Z(x)=e^{-2 \lambda}$ and $y^{2}(x)=e^{2 v}$ suggested by Durgapal and Bannerji [55] and with $c>0$ as arbitrary constant, the field equations (6)-(8) can be written as follows

$$
\begin{gathered}
\frac{\rho}{c}=-3 \dot{Z}-\frac{3(Z-1)(1-4 \beta \dot{Z})}{x} \\
\frac{p_{r}}{c}=\frac{3(Z-1)}{x}+\frac{6 Z \dot{y}}{y}-\frac{6 \beta(Z-1) Z \dot{y}}{x y} \\
\frac{p_{t}}{c}=4 Z[\beta(1-Z)+x] \frac{\ddot{y}}{y}+\left[\frac{2 \beta Z(1-Z)}{x}-2(x+\beta) \dot{Z}+6 Z-2 \beta Z \dot{Z}\right] \frac{\dot{y}}{y}+2\left[\frac{Z-1}{x}+\dot{Z}\right]
\end{gathered}
$$

where $\beta=4 \alpha c$ contains the Gauss-Bonnet coupling constant $\alpha$ and dots denote differentiation with respect to $x$. 
In this paper, we imposed the following equations of state, linear and quadratic, respectively, relating the radial pressure to the energy density, where $\gamma$ is a positive constant

$$
p_{r}=\gamma \rho \quad \text { and } \quad p_{r}=\gamma \rho^{2}
$$

\section{The New Anisotropic Models}

In this research, we take the form of the gravitational potential $Z(x)$ as $Z=(1-$ $a x)^{2}$ proposed for Thirukanesh and Ragel [27] and subsequently generalized by Malaver [37], taking as an arbitrary parameter. This potential is regular at the stellar center and well behaved in the interior of the sphere. Using $Z(x)$ in equation (9), we obtain

$$
\rho=c\left(12 a+48 a^{2} \beta-9(1+8 a \beta) a^{2} x+24 a^{4} \beta x^{2}\right)
$$

Substituting the equation (13) in the expression of the linear equation of state for the radial pressure (12), we have

$$
p_{r}=\gamma c\left(12 a+48 a^{2} \beta-9(1+8 a \beta) a^{2} x+24 a^{4} \beta x^{2}\right)
$$

With $Z(x)$ and (14) in equation (10), we have

$$
\frac{\dot{y}}{y}=\frac{3 a(2-a x)}{6(1-a x)^{2}[1+a \beta(2-a x)]}+\frac{\gamma\left[12 a+48 a^{2} \beta-9(1+8 a \beta) a^{2} x+24 a^{4} \beta x^{2}\right]}{6(1-a x)^{2}[1+a \beta(2-a x)]}
$$

Integrating equation (15) with respect to $y$, we obtain

$$
y(x)=c_{1}(a x-1)^{A}\left(a^{2} \beta x-2 a \beta-1\right)^{B} e^{\frac{c}{(a x-1)}}
$$

where the constants $A, B$ and $C$ are given by

$$
\begin{aligned}
& A=-\frac{8 a^{2} \beta^{2} \gamma+10 a \beta \gamma+3 \gamma}{2(a \beta+1)^{2}} \\
& B=-\frac{6 a \beta \gamma+5 \gamma-1}{2(a \beta+)^{2}} \\
& C=-\frac{\gamma-1}{2(a \beta+1)}
\end{aligned}
$$

and $c_{1}$ is the constant of integration. 
For the metric functions $e^{2 \lambda}$ and $e^{2 v}$, we have

$$
\begin{gathered}
e^{2 \lambda}=\frac{1}{(1-a x)^{2}} \\
e^{2 v}=c_{1}^{2}(a x-1)^{2 A}\left(a^{2} \beta x-2 a \beta-1\right)^{2 B} e^{\frac{2 C}{(a x-1)}}
\end{gathered}
$$

and the anisotropy can be written as

$$
\begin{aligned}
& \begin{array}{l}
\Delta=p_{t}-p_{r}=\left[4 \beta a x c(1-a x)^{2}(2-a x)\right. \\
\left.+4 x c(1-a x)^{2}\right]
\end{array}\left[\begin{array}{l}
\frac{\left(A^{2}-A\right) a^{2}}{(a x-1)^{2}}+\frac{2 A a^{3} B \beta}{(a x-1)\left(a^{2} \beta x-2 a \beta-1\right)} \\
-\frac{2 A a^{2} C}{(a x-1)^{3}}+\frac{B^{2} a^{4} \beta^{2}}{\left(a^{2} \beta x-2 a \beta-1\right)^{2}} \\
-\frac{B a^{4} \beta^{2}}{\left(a^{2} \beta x-2 a \beta-1\right)^{2}}-\frac{2 B a^{3} \beta C}{\left(a^{2} \beta x-2 a \beta-1\right)} \\
+\frac{2 C a^{2}}{(a x-1)^{3}}+\frac{C^{2} a^{2}}{(a x-1)^{4}}
\end{array}\right] \\
& +\left[4 a \beta c(1-a x)^{2}(a x-2)+4 a c(x+\beta)(1-a x)+4 a \beta(1-a x)^{3}\right]\left[\frac{A a}{a x-1}+\frac{B a^{2} \beta}{a^{2} \beta x-2 a \beta-1}\right. \\
& \left.-\frac{C a}{(a x-1)^{2}}\right]
\end{aligned}
$$

With the quadratic equation of state, we obtain for the radial pressure

$$
p_{r}=\gamma c^{2}\left(12 a+48 a^{2} \beta-9(1+8 a \beta) a^{2} x+24 a^{4} \beta x^{2}\right)^{2}
$$

and for the equation (10), we have $\quad \frac{\dot{y}}{y}=\frac{3 a(2-a x)}{6(1-a x)^{2}[1+a \beta(2-a x)]}+$ $\frac{\gamma c^{2}\left[12 a+48 a^{2} \beta-9(1+8 a \beta) a^{2} x+24 a^{4} \beta x^{2}\right]^{2}}{6(1-a x)^{2}[1+a \beta(2-a x)]}$

Integrating (24) with respect to $y$, we obtain

$$
y(x)=c_{2}\left(a^{2} \beta x-2 a \beta-1\right)^{D}(a x-1)^{E} e^{\frac{F x^{3}+G x^{2}+H x+}{(a x-1)}}
$$

Again the constants $D, E, F, G, H$ and $I$ are given by

$$
D=-\frac{108 a^{2} \beta^{2} c^{2} \gamma+180 a \beta c^{2} \gamma+75 c^{2} \gamma-\beta}{2(a \beta+1)^{2} \beta}
$$




$$
\begin{aligned}
E & =-\frac{48 a^{3} \beta^{2} c^{2} \gamma+63 a^{2} \beta c^{2} \gamma+18 a c^{2} \gamma+1}{2(a \beta+1)^{2}} \\
F & =-\frac{96 a^{6} \beta^{2} c^{2} \gamma+96 a^{5} \beta c^{2} \gamma}{2(a \beta+1)} \\
G & =-\frac{48 a^{3} c^{2} \gamma-432 a^{4} \beta c^{2} \gamma-480 a^{5} \beta^{2} c^{2} \gamma}{2(a \beta+1)} \\
H & =-\frac{384 a^{4} \beta^{2} c^{2} \gamma+336{ }^{3} \beta c^{2} \gamma-48 a^{2} c^{2} \gamma}{2(a \beta+1)} \\
I & =-\frac{3 a c^{2} \gamma+1}{2(a \beta+1)}
\end{aligned}
$$

$c_{2}$ is the constant of integration.

For the metric functions, we have $e^{2 \lambda}$ and $e^{2 v}$

$$
\begin{gathered}
e^{2 \lambda}=\frac{1}{(1-a x)^{2}} \\
e^{2 v}=c_{2}^{2}\left(a^{2} \beta x-2 a \beta-1\right)^{2 D}(a x-1)^{2 E} e^{\frac{2\left(F x^{3}+G x^{2}+H x+I\right)}{(a x-1)}}
\end{gathered}
$$

For the anisotropy $\Delta$, we have

$$
\begin{aligned}
& \Delta=p_{t}-p_{r} \\
& =\left[4 \beta a x c(1-a x)^{2}(2-a x)\right. \\
& {\left[\frac{\left(D^{2}-D\right) a^{4} \beta^{2}}{\left(a^{2} \beta x-2 a \beta-1\right)^{2}}+\frac{2 D a^{3} \beta E}{(a x-1)\left(a^{2} \beta x-2 a \beta-1\right)}\right.} \\
& +\frac{2 D a^{2} \beta}{\left(a^{2} \beta x-2 a \beta-1\right)}\left[\frac{3 F x^{2}+2 G x+H}{a x-1}-\frac{\left(F x^{3}+G x^{2}+H x+I\right) a}{(a x-1)^{2}}\right] \\
& \left.+4 x c(1-a x)^{2}\right]+\frac{\left(E^{2}-E\right) a^{2}}{(a x-1)^{2}}+\frac{2 E a}{(a x-1)}\left[\frac{3 F x^{2}+2 G x+H}{a x-1}-\frac{\left(F x^{3}+G x^{2}+H x+I\right) a}{(a x-1)^{2}}\right] \\
& +\frac{6 F x+2 G}{(a x-1)}-\frac{2\left(3 F x^{2}+2 G x+H\right) a}{(a x-1)^{2}}+\frac{2\left(F x^{3}+G x^{2}+H x+I\right) a^{2}}{(a x-1)^{3}} \\
& {\left[\frac{3 F x^{2}+2 G x+H}{a x-1}-\frac{\left(F x^{3}+G x^{2}+H x+I\right) a}{(a x-1)^{2}}\right]^{2}} \\
& +\left[4 a \beta c(1-a x)^{2}(a x-2)+4 a c(x+\beta)(1-a x)+4 a \beta(1-a x)^{3}\right]\left[\frac{D a^{2} \beta}{a^{2} \beta x-2 a \beta x-1}\right. \\
& \left.+\frac{3 F x^{2}+2 G x+H+E a}{a x-1}-\frac{\left(F x^{3}+G x^{2}+H x+I\right) a}{(a x-1)^{2}}\right] \\
& -4 a c(1-a x)-2 a c(a x-2)
\end{aligned}
$$




\section{Physical Acceptability in EGB Gravity}

For a model to be physically acceptable in EGB gravity, the following conditions should be satisfied [10,53]:

(i) The metric potentials $e^{2 \lambda}$ and $e^{2 v}$ assume finite values throughout the stellar interior and are singularity-free at the center $r=0$.

(ii) The energy density $\rho$ and the radial pressure $p_{r}$ should be positive inside the star.

(iii) The anisotropy is zero at the center $r=0$, i.e. $\Delta(r=0)=0$.

(iv) The energy density and radial pressure are decreasing functions with the radial parameter, i.e. $\frac{d p_{r}}{d r} \leq 0$ and $\frac{d \rho}{d r} \leq 0$ both in EGB gravity.

(v) Any physically acceptable model must satisfy the causality condition, that is, for the radial sound speed $v_{s r}^{2}=\frac{d p_{r}}{d \rho}$,we should have $0 \leq v_{s r}^{2} \leq 1$.

(vi) The boundary of the star defined by $r=R$ should be matched with the Einstein - GaussBonnet- Schwarzschild exterior solution given by

$$
d s^{2}=-F(r) d t^{2}+\frac{d r^{2}}{F(r)}+r^{2}\left(d \vartheta^{2}+\sin ^{2} \vartheta d \phi^{2}+\sin ^{2} \theta \sin ^{2} \phi d \psi^{2}\right)
$$

where $R$ is the radius of the star and

$$
F(r)=1+\frac{r^{2}}{4 \alpha}\left(1-\sqrt{1+\frac{8 M \alpha}{r^{4}}}\right)
$$

In Equation. (36),$M$ is associated with the gravitational mass of the hypersphere.

\section{Physical Features of the New Models}

For the linear equation of state, the metric potentials $e^{2 \lambda}$ and $e^{2 v}$ have finite values and remain positive throughout the stellar interior. At the center $e^{2 \lambda(0)}=1, e^{2 v(0)}=$ $c_{1}^{2}(-1)^{2 A}(-2 a \beta-1)^{2 B} e^{-2 C}$. We show that in $r=0,\left(e^{2 \lambda(r)}\right)_{r=0}^{\prime}=\left(e^{2 v(r)}\right)_{r=0}^{\prime}=0$ and it is verified that the gravitational potentials are regular at the center.

The energy density and radial pressure are positive and well behaved in the stellar interior. The obtained central density are $\rho(r=0)=a c(12+48 a \beta)$ and $p_{r}(r=0)=$ $\gamma c\left(12 a+48 a^{2} \beta\right)$, both positive if $a, \beta, c>0$.

In the surface of the fluid sphere $r=R$, we have $p_{r}(r=R)=0$ and is obtained for the radius of the star 


$$
R=\frac{\sqrt{\beta c\left(24 a \beta+\quad \sqrt{64 a^{2} \beta^{2}+16 a \beta+9}\right)}}{4 a \beta c}
$$

Differentiating Eq. (13) and Eq. (14), the expressions for density and radial pressure gradient are given by

$$
\begin{aligned}
& \frac{d \rho}{d r}=3 a^{2} c r\left[-6(1+8 a \beta)+32 a^{2} \beta c r^{2}\right] \\
& \frac{d p_{r}}{d r}=3 \gamma c^{2} a^{2} r\left[-6(1+8 a \beta)+32 a^{2} \beta c r^{2}\right]
\end{aligned}
$$

The radial sound speed for this model of compact star is obtained as

$$
v_{s r}^{2}=\frac{d p_{r}}{d \rho}=\gamma
$$

For the physically acceptability of the model of anisotropic star, it should satisfy the causality condition, i.e. $0 \leq v_{s r}^{2} \leq 1$.

Using the first fundamental form that consist in the continuity of the metric functions and their derivatives across the boundary $r=R$, we have

$$
\begin{aligned}
& \frac{1}{\left(1-a c R^{2}\right)^{2}}=\frac{1}{1+\frac{R^{2}}{4 \alpha}\left(1-\sqrt{1+\frac{8 \alpha M}{R^{4}}}\right)} \\
& c_{1}^{2}\left(a c R^{2}-1\right)^{2 A}\left(a^{2} \beta c R^{2}-2 a \beta-1\right)^{2 B} e^{\frac{2 C}{\left(a c R^{2}-1\right)}}=1+\frac{R^{2}}{4 \alpha}\left(1-\sqrt{1+\frac{8 \alpha}{R^{4}}}\right) \\
& \left.\frac{C a c}{\left(a c R^{2}-1\right)^{2}}\right] C_{1}^{2}\left(a c R^{2}-1\right)^{2 A}\left(a^{2} \beta c R^{2}-2 a \beta-1\right)^{2 B} e^{\frac{2 C}{a c c^{2}-1}} \\
& =-\frac{1}{2 \alpha}\left[\frac{1-\sqrt{1+\frac{8 \alpha M}{R^{4}}}}{\sqrt{1+\frac{8 \alpha M}{R^{4}}}}\right]
\end{aligned}
$$

and from the second fundamental form, we obtain

$$
12 a+48 a^{2} \beta-9(1+8 a \beta) a^{2} c R^{2}+24 a^{4} \beta c^{2} R^{4}=0
$$

The equations (41-44) are the conditions that allow determining the parameters a, A, B, C that describe the model. 
With the quadratic equation of state, we have for the metric potentials $e^{2 \lambda(0)}=1$, $e^{2 v(0)}=c_{2}^{2}(-2 a \beta-1)^{2 D}(-1)^{2 E} e^{-2 I}$. Again in $r=0$, it satisfies the condition $\left(e^{2 \lambda(r)}\right)_{r=0}^{\prime}=\left(e^{2 v(r)}\right)_{r=0}^{\prime}=0$ verifying that also the gravitational potentials are singularity- free at the origin.

The radial pressure is positive throughout the fluid configuration and the central radial pressure is $p_{r}(r=0)=\gamma c^{2}\left(12 a+48 a^{2} \beta\right)^{2}$, and is positive if $a, \beta, \gamma, c>0$. For the radial pressure gradient, we have

$$
\frac{d p_{r}}{d r}=2 \gamma c^{2}\left[12 a+48 a^{2} \beta-9(1+8 a \beta) a^{2} c r^{2}+24 a^{4} \beta c^{2} r^{4}\right]\left[-18 a^{2} c r(1+8 a \beta)+96 a^{4} \beta c^{2} r^{3}\right]
$$

For this case, the causality condition $0 \leq v_{s r}^{2} \leq 1$ implies that

$$
v_{s r}^{2}=\frac{d p_{r}}{d \rho}=2 \gamma c\left[12 a+48 a^{2} \beta-9(1+8 a \beta) a^{2} c r^{2}+24 a^{4} \beta c^{2} r^{4}\right]
$$

Again, with the first fundamental form, we can obtain

$$
\begin{aligned}
& \frac{1}{\left(1-a c R^{2}\right)^{2}}=\frac{1}{1+\frac{R^{2}}{4 \alpha}\left(1-\sqrt{1+\frac{8 \alpha M}{R^{4}}}\right)} \\
& c_{2}^{2}\left(a^{2} \beta c R^{2}-2 a \beta-1\right)^{2 D}\left(a c R^{2}-1\right)^{2 E} e^{\frac{2 F c^{3} R^{6}+2 G c^{2} R^{4}+H c R^{2}+1}{a c R^{2}-1}}=1+\frac{R^{2}}{4 \alpha}\left(1-\sqrt{1+\frac{8 \alpha M}{R^{4}}}\right) \\
& {\left[\begin{array}{l}
\frac{4 E a c}{a c R^{2}-1}+\frac{4 D a^{2} \beta c}{a^{2} \beta c R^{2}-2 a \beta-1} \\
\frac{12 F{ }^{3} R^{4}+8 G c^{2} R^{2}+2 H C}{a c R^{2}-1} \\
\frac{2\left(2 F c^{3} R^{6}+2 G c^{2} R^{4}+H c R^{2}\right) a c}{\left(a c R^{2}-1\right)^{2}}
\end{array}\right] C_{2}^{2}\left(a^{2} \beta c R^{2}-2 a \beta-1\right)^{2 D}\left(a c R^{2}-1\right)^{2 E} e^{\frac{2 F c^{3} R^{6}+2 G c^{2} R^{4}+H c R^{2}+1}{a c R^{2}-1}}} \\
& =-\frac{1}{2 \alpha}\left[\frac{1-\sqrt{1+\frac{8 \alpha M}{R^{4}}}}{\sqrt{1+\frac{8 \alpha M}{R^{4}}}}\right]
\end{aligned}
$$

and for the second fundamental form

$$
\left(12 a+48 a^{2} \beta-9(1+8 a \beta) a^{2} c R^{2}+24 a^{4} \beta c^{2} R^{4}\right)^{2}=0
$$

From the equation (44) and considering some particular cases for the Gauss-Bonnet coupling constant, we can calculate the corresponding values of the parameter $a$. For $\alpha=1$, 2,3 , we obtain the values $a=0.035 ; 0.0337 ; 0.03234$, respectively. In all the cases was it has 
been considered the radius $R=5.7 \mathrm{Km}$ and $c=1$. The Figures 1 and 2 present the dependency of $\rho$ and $\frac{d \rho}{d r}$ with the radial coordinates, respectively.

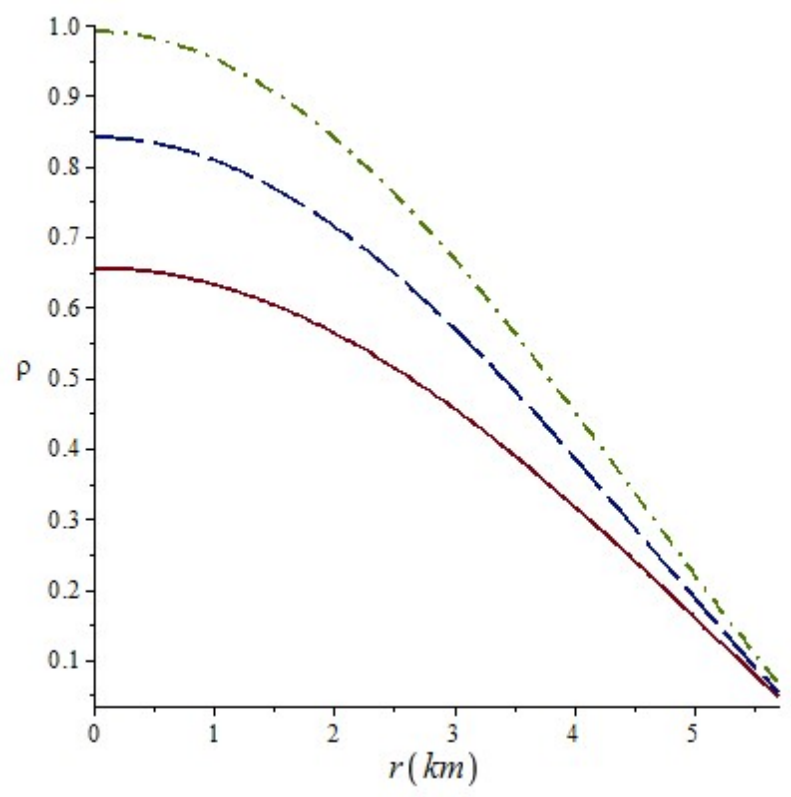

Figure 1. Energy density against radial parameter for $a=0.035$ and $\alpha=1$ (solid line); $a=0.0337$ and $\alpha=2$ (long-dash line); $a=0.03234$ and $\alpha=3$ (dashdot line).

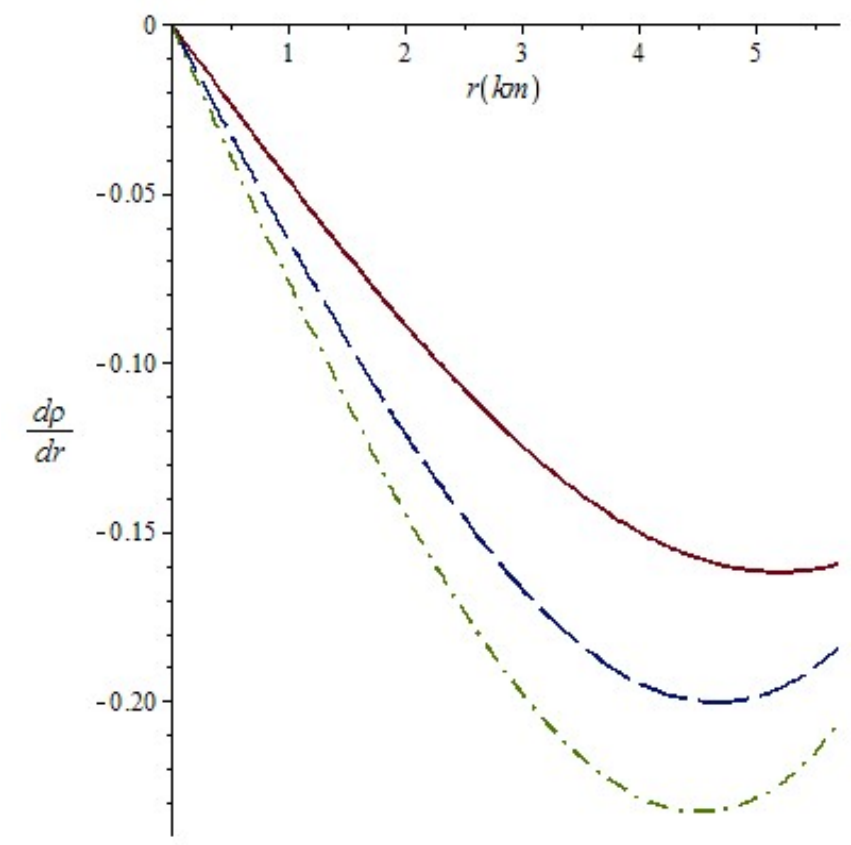

Figure 2. Energy density gradient against radial parameter for $a=0.035$ and $\alpha=1$ (solid line); $a=0.0337$ and $\alpha=2$ (long-dash line); $a=0.03234$ and $\alpha=3$ (dashdot line). 
The Figures 3,4 and 5 show the dependence of $p_{r}, \frac{d p_{r}}{d r}$, $\Delta$,respectively with the radial parameter in the linear regimen for the different values of coupling constant $\alpha$. In all the cases, it has been considered $R=5.7 \mathrm{Km}, c=1$ and $\gamma=1 / 3$.

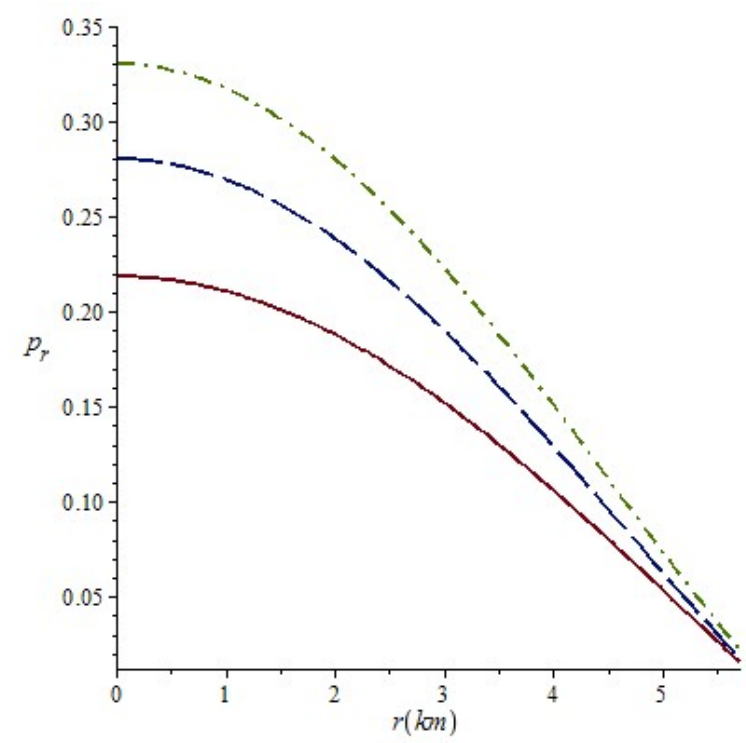

Figure 3. Radial pressure against radial parameter in linear regimen for $a=0.035$ and $\alpha=1$ (solid line); $a=0.0337$ and $\alpha=2$ (long-dash line); $a=0.03234$ and $\alpha=3$ (dashdot line).

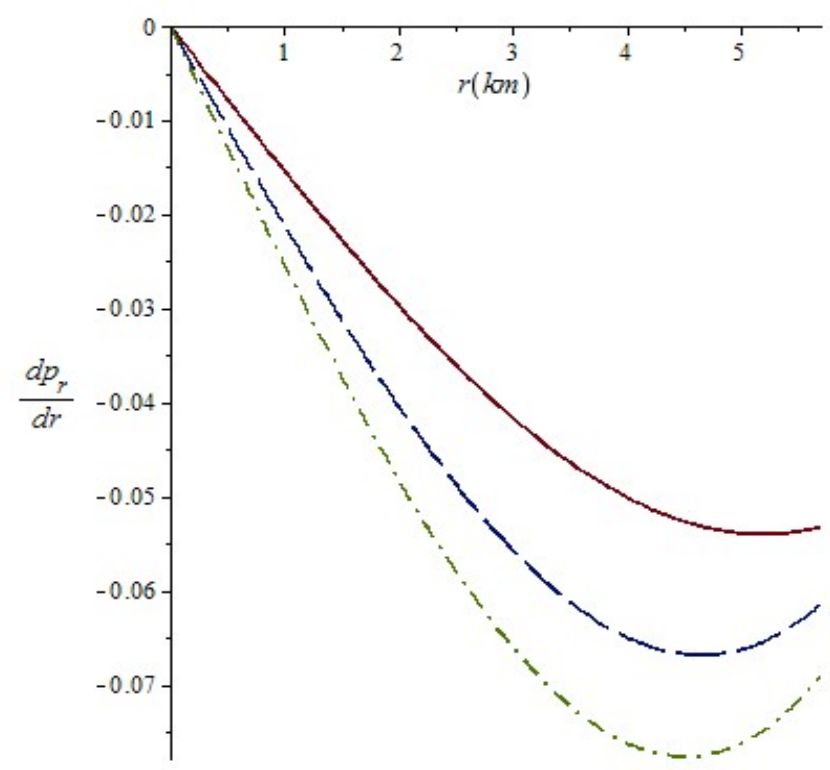

Figure 4. Radial pressure gradient against radial parameter in linear regimen for $a=$ 0.035 and $\alpha=1$ (solid line); $a=0.0337$ and $\alpha=2$ (long-dash line); $a=0.03234$ and $\alpha=3$ (dashdot line). 


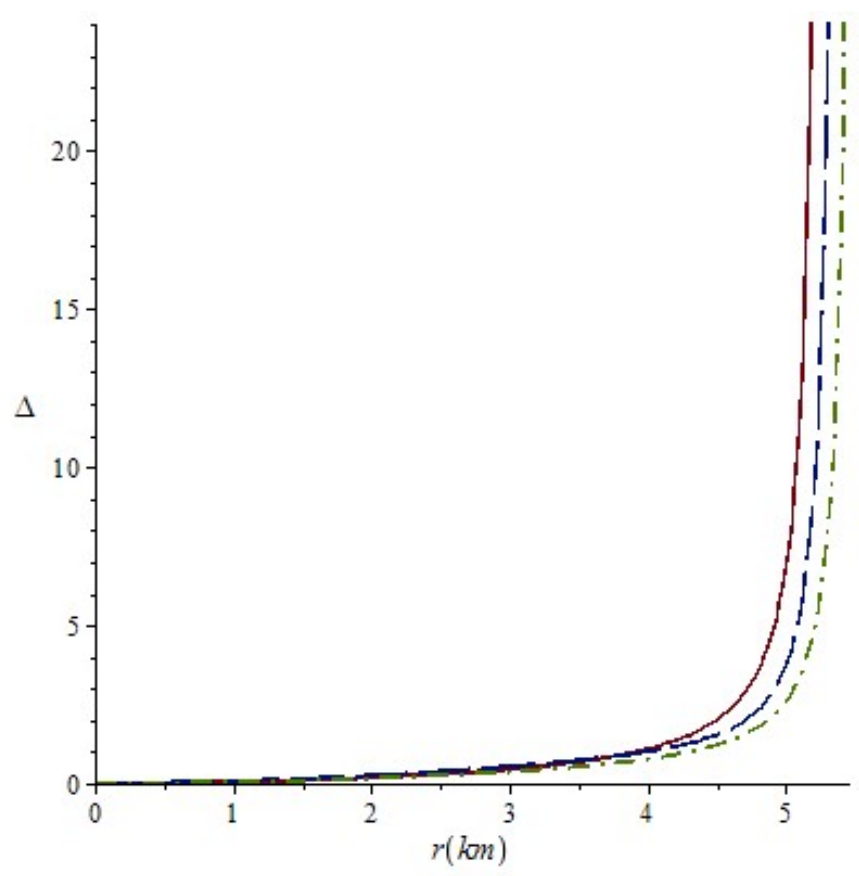

Figure 5. Anisotropy against radial parameter in linear regimen for $a=0.035$ and $\alpha=1$ (solid line); $\quad a=0.0337$ and $\alpha=2$ (long-dash line); $a=0.03234$ and $\alpha=3$ (dashdot line).

In two cases, linear and quadratic, the energy density remains positive, continuous and is monotonically decreasing function throughout the stellar interior (Figure 1). It is also noted that the density increases with increasing $\alpha$. The radial variation of energy density gradient has been shown in Figure 2, in which it is observed that $\frac{d \rho}{d r}<0$ in EGB gravity.

In the linear regimen, the radial pressure showed the same behavior by the energy density, that is, it is growing within the star and vanishes at a greater radial distance, but takes the higher values when $\alpha$ is increased and its results are shown in Figure 3. Again ,according to Figure 4, the profile of $\frac{d p_{r}}{d r}$ shows that radial pressure gradient is negative inside the stellar interior. The anisotropic factor is plotted in Figure 5 and it shows that vanishes at the centre of the star, i.e. $\Delta(r=0)=0$ [30]. We can also note that $\Delta$ admits lower values when $\alpha$ increases.

The Figures 6,7,8 and 9 show the dependence of $p_{r}, \frac{d p_{r}}{d r}, v_{s r}^{2}$ and $\Delta$ respectively with the radial coordinates in the quadratic case for the different values of coupling constant $\alpha$. In all the cases, it has been considered $R=5.7 \mathrm{Km}, c=1$ and $\gamma=1 / 3$. 


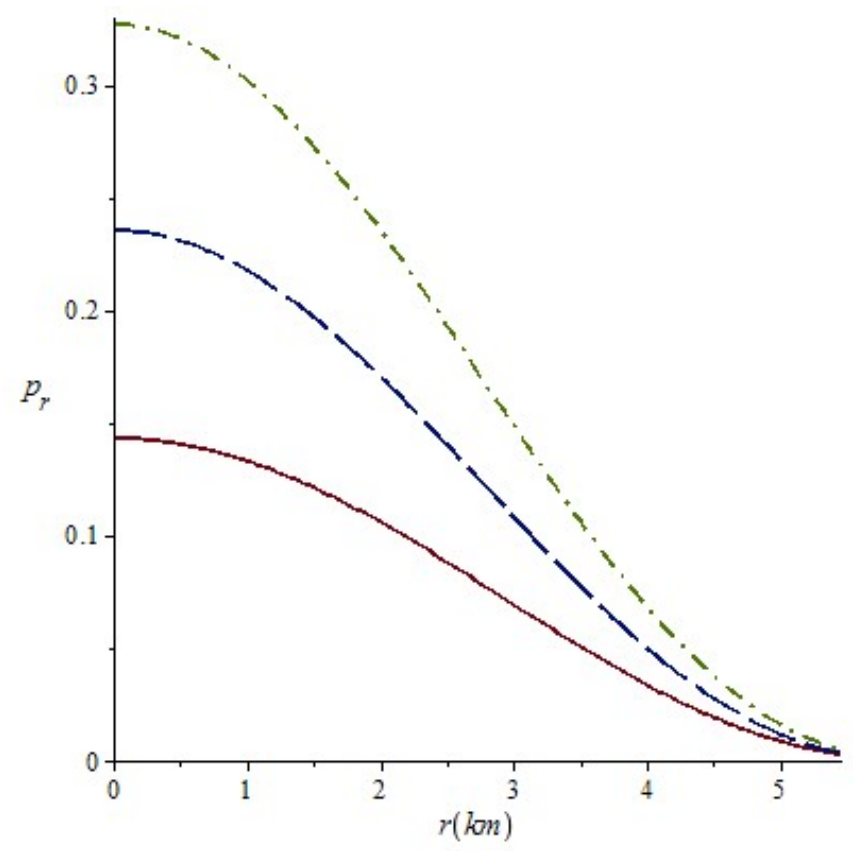

Figure 6. Radial pressure against radial parameter in quadratic regimen for $a=$ 0.035 and $\alpha=1$ (solid line); $a=0.0337$ and $\alpha=2$ (long-dash line); $a=0.03234$ and $\alpha=3$ (dashdot line).

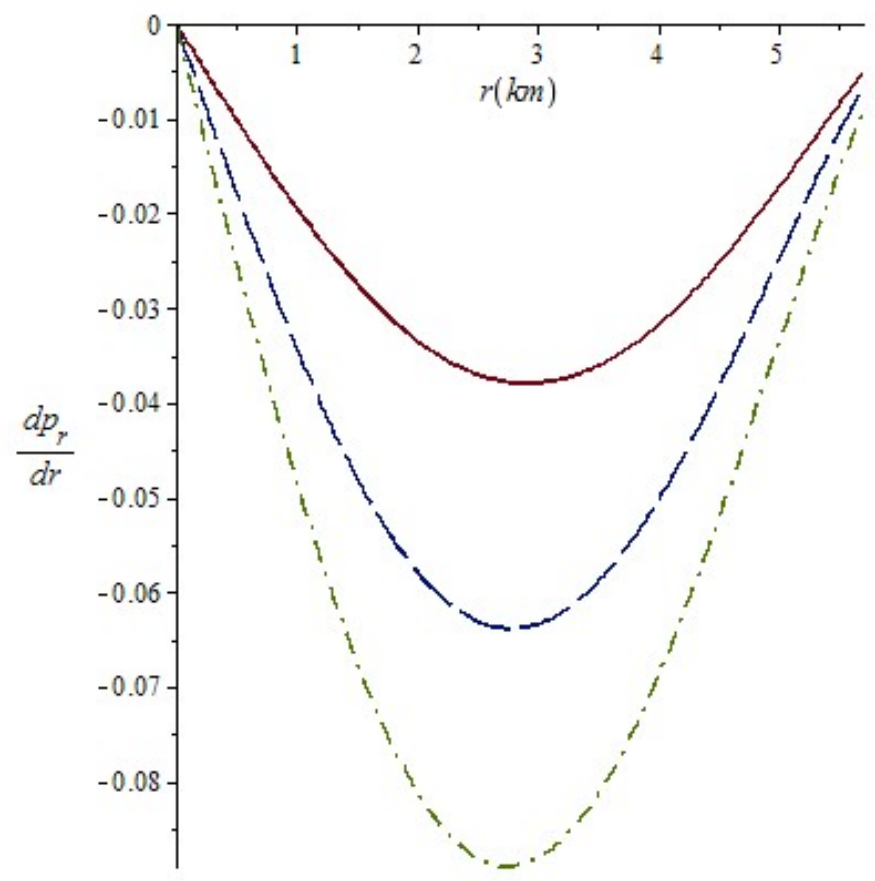

Figure 7. Radial pressure gradient against radial parameter in quadratic regimen for $a=$ 0.035 and $\alpha=1$ (solid line); $a=0.0337$ and $\alpha=2$ (long-dash line); $a=0.03234$ and $\alpha=3$ (dashdot line). 


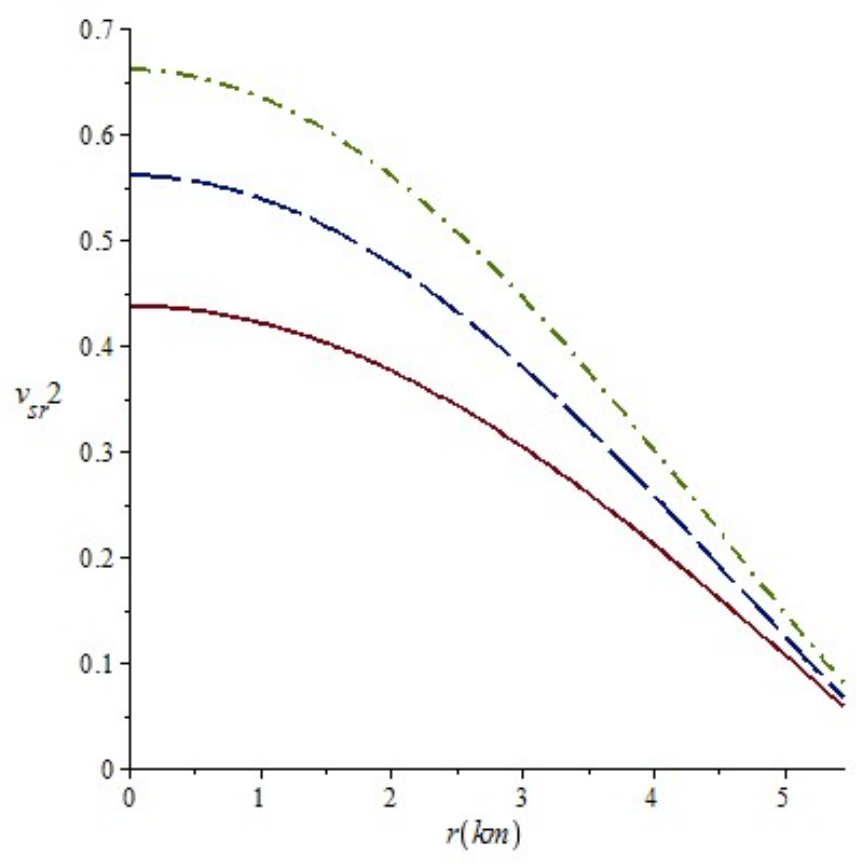

Figure 8. Radial sound speed against radial parameter in quadratic regimen for $a=0.035$ and $\alpha=1$ (solid line); $a=0.0337$ and $\alpha=2$ (long-dash line); $a=0.03234$ and $\alpha=3$ (dashdot line).

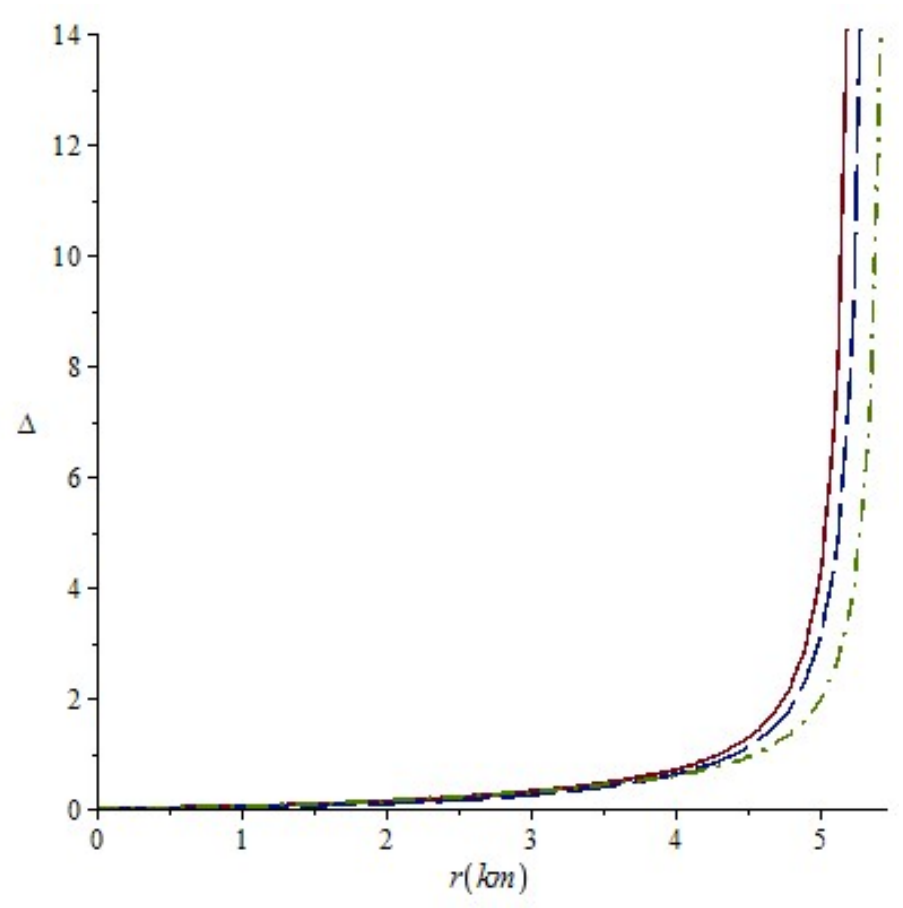

Figure 9. Anisotropy against radial parameter in quadratic regimen for $a=0.035$ and $\alpha=1$ (solid line); $a=0.0337$ and $\alpha=2$ (long-dash line); $a=0.03234$ and $\alpha=3$ (dashdot line). 
As in the linear regimen, with the quadratic equation, the radial pressure always is positive inside the star and vanishes at a finite radial distance and its results are shown in Figure 6. Again, the radial pressure increases when $\alpha$ takes higher values. In the Figure 7, it is also

verified that the gradient $\frac{d p_{r}}{d r}$ is negative in the stellar interior.

A physically acceptable model must satisfy the causality condition, i.e., the radial sound speed must be within the range $0 \leq v_{s r}^{2} \leq 1$. The profile of radial speed sound is plotted in Figure 8 for different values of coupling constant $\alpha$. In all the cases $v_{s r}^{2}$ is in the expected range and is a monotonic decreasing function with the radial coordinates. Figure 9 shows that the anisotropy is zero at the center $r=0$ and its value increases towards the surface of the star. As in the linear case $\Delta$ takes lower values when $\alpha$ increases.

\section{Conclusions}

In this paper, we have generated new models of compact stars within the framework of Einstein-Gauss-Bonnet gravity. With the use of Thirukkanesh-Ragel-Malaver ansatz for the gravitational potentials and with a linear and quadratic equation of state, we are able to produce two classes of exact solutions of the EGB field equations. We show that the developed configuration obeys the rigorous conditions required for the physical viability of the stellar model. It is to be noted in EGB gravity that the coupling constant $\alpha$ has nonnegligible effects on the physical quantities such as energy density and radial pressure of the star which increases with an increase in $\alpha$. As expected, the matching conditions require that the radial pressure vanishes at some finite radius of the stellar object and this defines the boundary of the star.

An evidence to the effect of the coupling constant can be observed in the behavior of energy density. In the two studied cases, linear and quadratic, when the Gauss-Bonnet constant increases, the energy density also increases and it allows that these models can support more masses. It is also noted that for all the values of the coupling constant $\alpha, v_{s r}^{2}$ is maximum at the centre and it decreases radially. Also, it is observed that inside of the star $0 \leq v_{s r}^{2} \leq 1$, which shows that the models are stable. Within the framework of EGB gravity, it is plausible to consider that the proposed models can describe real compact objects such as white dwarfs, neutron stars and pulsars.

\section{References}

[1] Kuhfitting, P.K.(2011). Some remarks on exact wormhole solutions, Adv. Stud. Theor. Phys. 2011, 5, 365.

[2] Bicak, J. Einstein equations: exact solutions, Encyclopaedia of Mathematical Physics. 2006,2, 165. 
[3] Malaver, M. Black Holes, Wormholes and Dark Energy Stars in General Relativity. Lambert Academic Publishing, Berlin. ISBN: 978-3-659-34784-9, 2013.

[4] Delgaty.;M.S.R.; Lake, K. Physical Acceptability of Isolated, Static, Spherically Symmetric, Perfect Fluid Solutions of Einstein's Equations. Comput. Phys. Commun. $1998,115,395$.

[5] Schwarzschild, K. Uber das Gravitationsfeld einer Kugel aus inkompressibler Flussigkeit nach der Einsteinschen Theorie. Math.Phys.Tech, 1916, 424-434.

[6] Tolman, R.C. Static Solutions of Einstein's Field Equations for Spheres of Fluid. Phys. Rev. 1939, 55, 364-373.

[7] Oppenheimer, J.R. and Volkoff, G. On Massive Neutron Cores. Phys. Rev. 1939, 55, 374-381.

[8] Chandrasekhar, S. The Maximum mass of ideal white dwarfs. Astrophys. J. 1931, 74, 81-82.

[9] Bhar P.; Rahaman F.; Ray S.; Chatterjee V. Possibility of higher-dimensional anisotropic compact star. Eur. Phys. J. C. 2015; 75(5):190.

[10] Bhar, P.; Govender, M. Charged compact star model in Einstein-Maxwell-Gaussgravity. Astrophys Space Sci. 2019, 364,186. https://doi.org/10.1007/s10509-019-3675-0

[11] Gupta, Y.K.; Maurya, S.K. A class of charged analogues of Durgapal and Fuloria superdense star. Astrophys. Space Sci. 2011, 331, 135-144.

[12] Kiess, T.E. Exact physical Maxwell-Einstein Tolman-VII solution and its use in stellar models . Astrophys. Space Sci. 2012, 339, 329-338.

[13] Mafa Takisa, P.; Maharaj, S.D. Some charged polytropic models. Gen.Rel.Grav. 2013, 45, 1951-1969.

[14] Malaver, M.; Kasmaei, H.D. Relativistic stellar models with quadratic equation of state. International Journal of Mathematical Modelling \& Computations. 2020, 10, 111-124.

[15] Malaver, M. New Mathematical Models of Compact Stars with Charge Distributions. International Journal of Systems Science and Applied Mathematics. 2017, 2, 93-98, DOI: $10.11648 / \mathrm{j} . \mathrm{ijssam} .20170205 .13$.

[16] Malaver, M. Generalized Nonsingular Model for Compact Stars Electrically Charged. World Scientific News. 2018, 92, 327-339.

Available online: http://www.worldscientificnews.com/wpcontent/uploads/2017/ 12/WSN-922-2018-327-339.pdf 
[17] Ivanov, B.V. Static charged perfect fluid spheres in general relativity. Phys. Rev.D65. 2002, 104011.

[18] Sunzu, J.M.; Maharaj, S.D.; Ray, S. Quark star model with charged anisotropic matter. Astrophysics. Space.Sci. 2014, 354, 517-524.

[19] Sunzu,J.M. Realistic Polytropic Models for Neutral Stars with Vanishing Pressure Anisotropy. Global Journal of Science Frontier Research: A Physics and Space Science. 2018, 18, ISSN 2249-4626.

https://journalofscience.org/index.php/GJSFR/article/view/2168

[20] Komathiraj, K.; Maharaj, S.D. Analytical models for quark stars. Int. J. Mod. Phys. 2007, D16, 1803-1811.

[21] Malaver, M. Analytical models for compact stars with a linear equation of state. World Scientific News, 2016, 50, 64-73.

[22] Bombaci, I. Observational evidence for strange matter in compact objects from the X- ray burster 4U 1820-30, Phys. Rev. 1997, C55, 1587- 1590.

[23] Thirukkanesh, S. and Maharaj, S.D. Charged anisotropic matter with a linear equation of state. Class. Quantum Gravity. 2008, 25, 235001.

[24] Dey, M.; Bombaci, I.;, Dey, J.; Ray, S.; Samanta, B.C. Strange stars with realistic quark vector interaction and phenomenological density-dependent scalar potential, Phys. Lett. 1998, B438, 123-128.

[25] Usov, V. V. Electric fields at the quark surface of strange stars in the color-flavor locked phase. Phys. Rev. D. 2004, 70, 067301, DOI:_10.1103/PhysRevD.70.067301.

[26] Feroze, T.; Siddiqui, A. Charged anisotropic matter with quadratic equation of state. Gen. Rel. Grav. 2011, 43, 1025-1035.

[27] Thirukkanesh, S.; Ragel, F.C. Exact anisotropic sphere with polytropic equation of state. PRAMANA-Journal of physics. 2012, 78, 687-696.

[28] Malaver, M. Analytical model for charged polytropic stars with Van der Waals Modified Equation of State. American Journal of Astronomy and Astrophysics. 2013, $1,37-42$.

[29] Tello-Ortiz, F.; Malaver, M.; Rincón, A.; Gomez-Leyton, Y. Relativistic Anisotropic Fluid Spheres Satisfying a Non-Linear Equation of State. Eur. Phys. J. C. 2020, 80, 371, https://doi.org/10.1140/epjc/s10052-020-7956-0. 
[30] Esculpi, M.; Malaver, M.; Aloma, E. A Comparative Analysis of the Adiabatic Stability of Anisotropic Spherically Symmetric solutions in General Relativity. Gen. Relat.Grav. 2007, 39, 633-652, DOI: 10.1007/s10714-007-0409-3.

[31] Cosenza M.; Herrera L.; Esculpi M.; Witten L. Evolution of radiating anisotropic spheres in general relativity. Phys.Rev.1982, D 25, 2527-2535.

[32] Herrera L. Cracking of self-gravitating compact objects. Phys. Lett. 1992, A165, 206-210.

[33] Herrera L.; Nuñez L. Modeling 'hydrodynamic phase transitions' in a radiating spherically symmetric distribution of matter. The Astrophysical Journal. 1989, 339, 339-353.

[34] Herrera L.; Ruggeri G. J.; Witten L. Adiabatic Contraction of Anisotropic Spheres in General Relativity. The Astrophysical Journal. 1979, 234, 1094-1099.

[35] Herrera L.; Jimenez L.; Leal L.; Ponce de Leon J.; Esculpi M.; Galina V. Anisotropic fluids and conformal motions in general relativity. J. Math. Phys. 1984, 25, 3274.

[36] Malaver, M. Quark Star Model with Charge Distributions. Open Science Journal of Modern Physics. 2014, 1, 6-11.

[37] Malaver, M. Strange Quark Star Model with Quadratic Equation of State. Frontiers of Mathematics and Its Applications. 2014, 1, 9-15.

[38] Malaver, M. Charged anisotropic models in a modified Tolman IV space time. World Scientific News. 2018, 101, 31-43.

[39] Malaver, M. Charged stellar model with a prescribed form of metric function $y(x)$ in a Tolman VII spacetime. World Scientific News.2018, 108, 41-52.

[40] Malaver, M. Classes of relativistic stars with quadratic equation of state. World Scientific News. 2016, 57, 70 -80.

[41] Sunzu, J.; Danford, P. New exact models for anisotropic matter with electric field. Pramana-J. Phys. 2017, 89, 44.

[42] Bowers, R.L.;Liang, E.P.T. Anisotropic Spheres in General Relativity. Astrophys. J. $1974,188,657-665$.

[43] Sokolov. A. I. Phase transitions in a superfluid neutron liquid. Sov. Phys. JETP. 1980, $52,575-576$. 
[44] Bhar P.; Murad MH.; Pant N. Relativistic anisotropic stellar models with Tolman VII spacetime. Astrophys Space Sci. 2015, 359: 13. doi: 10.1007/s10509-015-2462-9.

[45] Malaver, M. Some new models of anisotropic compact stars with quadratic equation of state. World Scientific News. 2018, 109, 180-194.

[46] Malaver, M. Charged anisotropic matter with modified Tolman IV potential. Open Science Journal of Modern Physics. 2015, 2(5), 65-71.

[47] Feroze T.; Siddiqui A. Some Exact Solutions of the Einstein-Maxwell Equations with a Quadratic Equation of State. J Korean Phys Soc. 2014, 65,944-947. doi: $10.3938 / \mathrm{jkps} .65 .944$

[48] Sen, B.; Grammenos, Th.; Bhar, P.; Rahaman, F. Mathematical modeling of compact anisotropic relativistic fluid spheres in higher spacetime dimensions. Math. Meth. Appl. Sci. 2016, 41, 1062-1067. doi: https://doi.org/10.1002/mma.4128

[49] Kaluza T. Unitätsproblem in der Physik. Sitz. Preuss. Acad. Wiss. 1921, Berlin, 966- 972.

[50] Klein O. Quantentheorie und fünfdimensionale Relativitätstheorie. Z. Physik.1926. $37,895-906$.

[51] Paul, B.C.; Dey, S. Relativistic Star in Higher dimensions with Finch and Skea Geometry. Astrophys Space Sci. 2018, 363,220. doi: https://doi.org/10.1007/s10509-018-3438-3.

[52] Bhar, P.; Singh, K.N.; Tello-Ortiz, F. Compact star in Tolman-Kuchowicz spacetime in background of Einstein-Gauss-Bonnet gravity. Eur. Phys. J. C.2019, 79, 922. doi: https://doi.org/10.1140/epjc/s10052-019-7438-4.

[53] Bhar, P.; Govender, M.; Sharma, R. A comparative study between EGB gravity and GTR by modeling compact stars. Eur. Phys. J. C. 2017, 77, 109.

[54] Horvath.; Moraes, P.H.R.S. Modelling a $2.5 M_{\odot}$ compact star with quark matter. International Journal of Modern Physics D, 2020. 30, 2150016.

[55] Durgapal, M.C.; Bannerji, R. New analytical stellar model in general relativity. Phys.Rev. D27. 1983, 328-331. 